Monatsh. Math. 130, 161-170 (2000)

Monatshefte für

Mathematik

(C) Springer-Verlag 2000

Printed in Austria

\title{
Quasi-Diagonal Behaviour and Smooth Weyl Sums
}

\author{
By \\ Trevor D. Wooley* \\ University of Michigan, Ann Arbor, MI, USA
}

(Received 13 September 1999)

\begin{abstract}
Estimates are provided for small moments of exponential sums over smooth numbers substantially sharper than available hitherto. These bounds arise from the author's recent breaking of "classical convexity" in Waring's problem. The methods underlying these new estimates provide guidance on good choices of parameters in the new iterative methods for smaller exponents.
\end{abstract}

1991 Mathematics Subject Classification: 11L15, 11L07, 11P55

Key words: Exponential sums, mean value estimates, smooth Weyl sums

\section{Introduction}

Even moments of exponential sums have a natural interpretation in terms of the number of solutions of associated diophantine equations. This simple observation underlies almost all of the progress achieved in the theory of the circle method since the pioneering work of Hardy and Littlewood. Rather recently, the author has devised an extension of Vaughan's new iterative method (see [15]; Vaughan's work is described in [11], [12]) which permits odd and fractional moments of exponential sums over smooth numbers to be estimated non-trivially, thereby breaking away from the bounds following from "classical convexity" (that is, bounds arising from the application of Hölder's inequality to interpolate between even moments). Moreover, the new methods permit substantially greater flexibility in the iterative methods for bounding mean values of smooth Weyl sums. On one hand, this flexibility leads to particularly sharp upper bounds for small moments of these exponential sums, close indeed to the lower bounds stemming from a consideration of diagonal solutions alone. On the other hand, when it comes to calculating upper bounds for mean values of smooth Weyl sums of smaller degree, this increased flexibility causes difficulty in obtaining an optimal choice of parameters, and explicit calculations therefore involve an inordinate expenditure of computational effort. Given the growing list of applications of these new bounds for smooth Weyl sums (see [1], [2], [3], [4], [5], [6]), the latter problem is not inconsequential. In response to this difficulty, the object of the present paper is to report on investigations concerning very small moments of smooth Weyl sums, and the choice of parameters in the associated application of the iterative method.

\footnotetext{
* Packard Fellow, and supported in part by NSF grant DMS-9970440
} 
These investigations lead to significantly sharper bounds than available hitherto for the latter moments, and offer heuristic guidance on where to seek optimal parameters in the iterative method.

In order to describe our conclusions, we must first introduce some notation. Let $P$ be a large real number, let $R$ be a real number with $2 \leqslant R \leqslant P$, and denote by $\mathscr{A}(P, R)$ the set of $R$-smooth numbers up to $P$, that is

$$
\mathscr{A}(P, R)=\{n \in[1, P] \cap \mathbb{Z}: p \text { prime and } p \mid n \Rightarrow p \leqslant R\} .
$$

Let $k$ be a fixed positive integer exceeding 2, and define the smooth Weyl sum $f(\alpha)=f(\alpha ; P, R)$ by

$$
f(\alpha ; P, R)=\sum_{x \in \mathscr{A}(P, R)} e\left(\alpha x^{k}\right),
$$

where $e(z)$ denotes $e^{2 \pi i z}$. Further, when $s$ is a positive real number, define the mean value $U_{s}(P, R)=U_{s}^{(k)}(P, R)$ by

$$
U_{s}(P, R)=\int_{0}^{1}|f(\alpha ; P, R)|^{s} d \alpha .
$$

We say that an exponent $\mu_{s}=\mu_{s, k}$ is permissible whenever the exponent has the property that for each $\varepsilon>0$, there exists a positive number $\eta=\eta(\varepsilon, s, k)$ such that whenever $R \leqslant P^{\eta}$, one has

$$
U_{s}(P, R) \ll_{\varepsilon, s, k} P^{\mu_{s, k}+\varepsilon} .
$$

Permissible exponents certainly exist, since for each $s$ the estimate $U_{s}(P, R) \ll P^{s}$ is trivial. It is convenient also to define an exponent $\delta_{s}=\delta_{s, k}$ to be associated whenever the exponent $\mu_{s}=\frac{1}{2} s+\delta_{s}$ is permissible.

In view of work of Hooley, Greaves and Skinner and Wooley concerning sums of two $k$ th powers (see [7], [8], [9], [10] for the sharpest available conclusions), when $k>2$ one knows that for each $\eta_{1}$ and $\eta_{2}$ with $\eta_{1}>\eta_{2}>0$, whenever $P^{\eta_{2}} \leqslant R \leqslant P^{\eta_{1}}$, one has

$$
P^{2} \ll \int_{0}^{1}|f(\alpha ; P, R)|^{4} d \alpha \ll P^{2} .
$$

As a consequence of Hölder's inequality, therefore, one readily deduces that under the same conditions on $R$, for each real number $s$ with $0<s \leqslant 4$,

$$
P^{s / 2} \ll U_{s}(P, R) \ll P^{s / 2} .
$$

On accounting for the underlying diagonal contribution, a formal application of the Hardy-Littlewood method suggests that when $k>2$, for each positive real number $s$, one should have

$$
P^{s / 2}+P^{s-k} \ll_{s, k} U_{s}(P, R) \ll_{s, k} P^{s / 2}+P^{s-k},
$$

whence, in particular, the inequalities (1) should hold for each real number $s$ with $0<s \leqslant 2 k$. While for $0<s \leqslant 4$, we have seen already that this conjectured 
conclusion does indeed hold, the sharpest conclusion currently available for intermediate values of $s$ is that

$$
\delta_{s, k}=\frac{8 k^{1 / 2}}{e s} \exp \left(-\frac{16 k}{e^{2} s^{2}}\right)
$$

is an associated exponent for $4<s \leqslant 4 e^{-1} k^{1 / 2}$ (see [15, Theorem 1.3]). Thus, when $\psi(k)$ is a monotonic increasing function of $k$ tending to infinity as $k$ tends to infinity, and $s$ is a real number with $4<s \leqslant k^{1 / 2} / \psi(k)$, then there exists a positive number $\eta=\eta(s, k)$ such that whenever $R \leqslant P^{\eta}$, one has

$$
U_{s}(P, R) \ll P^{s / 2+\delta_{s, k}},
$$

where $\delta_{s, k} \rightarrow 0$ as $k \rightarrow \infty$. We have previously referred to these asymptotically diagonal upper bounds as exhibiting quasi-diagonal behaviour (see the introduction of [14]).

It should be apparent from the above discussion that the smallest values of $s$ that retain interest are those close to 4 . The main conclusion of this paper, which we establish in $\S 3$ below, is that as $s$ converges to 4 from above, and independently as $k$ increases to infinity, the mean values $U_{s}^{(k)}(P, R)$ approach diagonal behaviour extremely rapidly.

Theorem 1. Let $k$ be an integer with $k \geqslant 60$, and let $\sigma$ be a positive number with $\sigma \leqslant 8 e /(k+1)$. Then the exponent $\delta_{4+\sigma}=\delta_{4+\sigma, k}$ is associated, where

$$
\delta_{4+\sigma}=\left(\frac{k \sigma}{24}\right)^{1+(k+1) /(2 e)}
$$

It follows, in particular, that whenever the hypotheses of Theorem 1 are satisfied, then one has

$$
\delta_{4+\sigma} \ll_{k} \sigma^{1+(k+1) /(2 e)} .
$$

For comparison, the conclusion of the corollary to [15, Theorem 1.1] yields an associated exponent

$$
\delta_{4+\sigma}=e \sigma^{\frac{\log (k+1)}{\log 2}} \delta_{6}
$$

When $k$ is large and $\sigma$ is sufficiently small in terms of $k$, the superiority of Theorem 1 is clear.

The condition in Theorem 1 that $\sigma \leqslant 8 e /(k+1)$ may give the impression that our methods are ineffective when $\sigma>8 e /(k+1)$. We therefore record a consequence of our methods concerning associated exponents $\delta_{4+\sigma}$ with $\sigma$ of intermediate size.

Theorem 2. Let $k$ be an integer with $k \geqslant 3$, and let $\sigma_{0}$ and $\sigma_{1}$ be positive numbers with

$$
\frac{8 e}{k+1} \leqslant \sigma_{1} \leqslant \frac{4 \sigma_{0}}{4+\sigma_{0}} \leqslant 2 .
$$


Then, whenever $\delta_{4+\sigma_{0}}$ is an associated exponent, one has that $\delta_{4+\sigma_{1}}$ is also associated, where

$$
\frac{\delta_{4+\sigma_{1}}}{\delta_{4+\sigma_{0}}}=e^{2}(k+1)\left(\frac{\sigma_{1}}{\sigma_{0}}\right)^{2+\left(4 / \sigma_{0}\right) \log \left((k+1) \sigma_{0} / 16\right)} .
$$

The conclusion of Theorem 1 shows that associated exponents $\delta_{4+\sigma}$ exist satisfying (2), at least, that is, when $k$ is large. We are also able to derive conclusions for smaller $k$, and these we record in the following theorem.

Theorem 3. When $k$ is an integer with $k \geqslant 8$, define the parameter $\xi=\xi(k)$ to be the real solution of the equation

$$
(1-\xi) \log (1-\xi)+\xi \log \xi+\xi \log \left(\frac{k+1}{2}\right)=0 .
$$

Define $\xi(k)$ to be $1 / 2$ when $3 \leqslant k \leqslant 7$. Further, for each integer $k$ with $k \geqslant 3$, define the exponent $\alpha=\alpha(k)$ by

$$
\alpha(k)= \begin{cases}\log (k+1) / \log 2, & \text { when } 3 \leqslant k \leqslant 7 \\ 1+1 / \xi(k), & \text { when } k \geqslant 8 .\end{cases}
$$

Finally, suppose that $\sigma_{0}$ and $\sigma_{1}$ are positive numbers with $\sigma_{1} \leqslant \sigma_{0}(1-\xi) \leqslant 4 \xi$. Then whenever $\delta_{4+\sigma_{0}}$ is an associated exponent, one has that $\delta_{4+\sigma_{1}}$ is also associated, where

$$
\delta_{4+\sigma_{1}}=e^{2}(k+1)\left(\frac{\sigma_{1}}{\sigma_{0}}\right)^{\alpha} \delta_{4+\sigma_{0}} .
$$

In particular, there is an associated exponent $\delta_{4+\sigma}$ satisfying $\delta_{4+\sigma} \ll_{k} \sigma^{\alpha}$.

We record in the table below the exponents $\alpha(k)$, for $3 \leqslant k \leqslant 20$, that arise in the statement of Theorem 3. The relevant data is recorded to five decimal places, with the final digit rounded down. For comparison, the corollary to Theorem 1.1 of [15] yields (3), which is of similar strength to the conclusion of Theorem 3 for $3 \leqslant k \leqslant 7$, but weaker for $k \geqslant 8$.

Table of exponents

$\begin{array}{ccccccccc}k & 2 \xi(k) & \alpha(k) & k & 2 \xi(k) & \alpha(k) & k & 2 \xi(k) & \alpha(k) \\ 3 & 1.00000 & 2.00000 & 9 & 0.84669 & 3.36213 & 15 & 0.57850 & 4.45721 \\ 4 & 1.00000 & 2.32192 & 10 & 0.78616 & 3.54399 & 16 & 0.54940 & 4.64031 \\ 5 & 1.00000 & 2.58496 & 11 & 0.73362 & 3.72620 & 17 & 0.52307 & 4.82351 \\ 6 & 1.00000 & 2.80735 & 12 & 0.68759 & 3.90868 & 18 & 0.49915 & 5.00678 \\ 7 & 1.00000 & 3.00000 & 13 & 0.64696 & 4.09136 & 19 & 0.47731 & 5.19013 \\ 8 & 0.91711 & 3.18074 & 14 & 0.61083 & 4.27422 & 20 & 0.45729 & 5.37353\end{array}$

Oversimplifying our argument considerably, the iterative method used to obtain the conclusion of Theorem 3 involves bounding the mean value $U_{s}(P, R)$ in the shape

$$
U_{s}(P, R) \ll\left(P^{\theta} R\right)^{s-3 t}\left(P^{t(1+\theta)} U_{s-2 t}\left(P^{1-\theta}, R\right)+T_{s, t}\right),
$$


where $t$ and $\theta$ are suitably chosen real numbers with $0 \leqslant \theta \leqslant 1 / k$ and $0<t \leqslant 1$, and

where

$$
T_{s, t}=\int_{0}^{1}\left|F(\alpha)^{t} f\left(\alpha ; P^{1-\theta}, R\right)^{s-2 t}\right| d \alpha,
$$

$$
F(\alpha)=\sum_{\substack{u \in \mathscr{A}\left(P^{\theta} R, R\right) \\ u>P^{\theta}}} \sum_{\substack{z_{1}, z_{2} \in \mathscr{A}(P, R) \\ z_{1} \equiv z_{2} \bmod u^{k} \\ z_{1} \neq z_{2}}} e\left(\alpha u^{-k}\left(z_{1}^{k}-z_{2}^{k}\right)\right) .
$$

The selection of the optimal parameter $t$ is one of the difficulties in the execution of this version of the new iterative method mentioned in the introduction. In the proof of Theorem 3, this parameter $t$ is equal to $2 \xi(k)$, and so it is apparent that when $s$ is very close to 4 , the parameter $t$ should be about $2 \xi(k)$. It is an empirical fact, based on extensive computations of workers in this area, that the optimal methods tend to be more biased in favour of the use of mean value estimates which are predominantly diagonal in nature for smaller values of $s$. Thus the analysis involved in the proof of Theorem 3 suggests strongly that optimal use of the iterative method for any value of $s$ will necessitate a choice for the parameter $t$ at least as large as $2 \xi(k)$. In particular, one should take $t=1$ for $3 \leqslant k \leqslant 7$.

Throughout, $k$ will be an integer exceeding 2 , and $s$ will be a positive real number. We use $\varepsilon$ and $\eta$ to denote sufficiently small positive numbers, and $P$ to denote a large positive number depending at most on $k, s, \varepsilon$ and $\eta$. The implicit constants in Vinogradov's well-known notation, $\ll$ and $\gg$, will depend at most on $k, s, \varepsilon$ and $\eta$. Also, we write $[x]$ for the greatest integer not exceeding $x$. We adopt the following convention concerning the numbers $\varepsilon$ and $R$. Whenever $\varepsilon$ or $R$ appear in a statement, either implicitly or explicitly, we assert that for each $\varepsilon>0$, there exists a positive number $\eta(\varepsilon, s, k)$ such that the statement holds whenever $R \leqslant P^{\eta}$. Note that the "value" of $\varepsilon$, and $\eta$, may change from statement to statement, and hence also the dependency of implicit constants on $\varepsilon$ and $\eta$. We observe that since our iterative methods will involve only a finite number of statements (depending at most on $k, s$ and $\varepsilon$ ), there is no danger of losing control of implicit constants through the successive changes in our arguments.

\section{Mean Value Estimates for Smooth Weyl Sums}

A discussion of the themes underlying the calculation of permissible exponents $\mu_{s}$ may be found in [15]. The particular cases of cubic and biquadratic smooth Weyl sums are discussed in detail in [1, §2], [4] and [6], respectively. In order to provide a basis for the methods of interest to us within this paper, we return to the methods of [15], taking the opportunity to update the latter in the light of subsequent developments.

Lemma 1. Let $k$ be an integer with $k \geqslant 3$, and define the integer $\tau=\tau(k)$ to be 1 when $k=3$, and to be 0 otherwise. Let $u$ and $t$ be real numbers with $0<t \leqslant 1$ and $u+2 t>4$. Also, let $v$ be a real number with

$$
\frac{u}{1-t / 4} \leqslant v \leqslant \frac{u}{1-t / 2}
$$


and write $w=1-u / v$. Finally, suppose that $\mu_{u, k}$ and $\mu_{v, k}$ are permissible exponents. Then the exponent $\mu_{u+2 t, k}$ is permissible, where

$$
\mu_{u+2 t}=\mu_{u}(1-\theta)+t+u \theta
$$

and

$$
\theta=\frac{t / 2-w+(1-w) \mu_{v}-\mu_{u}}{k(t-w)+w-\tau(t / 2-w)+(1-w) \mu_{v}-\mu_{u}} .
$$

Proof. Suppose that $u$ and $v$ satisfy the hypotheses of the statement of the lemma, and write $s=u+2 t$. Take $\phi$ to be a real number with $0 \leqslant \phi \leqslant 1 / k$ to be chosen later, and write

$$
M=P^{\phi}, \quad H=P M^{-k} \text { and } Q=P M^{-1} .
$$

On following the argument of [15, $\S 4]$ surrounding equations (4.2) and (4.3) of that paper, we find that our choice for $\phi$ is determined from the equation

$$
(P M)^{t} Q^{\mu_{u}}=P^{t / 2} H^{t-w} M^{t-w+\tau(t / 2-w)} Q^{(1-w) \mu_{v}} .
$$

Here we note that our definition of $\tau(k)$ differs from that employed in [15] in that $\tau(k)$ is now 0 even in the cases in which $k \geqslant 8$ and $k$ is even, whereas in the latter source one has $\tau(k)=1$ in these circumstances. On inspecting [15, §4], and examining the proof of [15, Lemma 3.4], one finds that this enhanced definition of $\tau(k)$ will be justified on establishing an estimate of the shape

$$
\sum_{1 \leqslant e \leqslant P} \int_{0}^{1}\left|F_{d, e}(\alpha)\right|^{4} d \alpha \ll d^{A} P^{2+\varepsilon}(M H)^{3},
$$

for a suitable positive number $A=A(k)$, in which we write

$$
F_{d, e}(\alpha)=\sum_{1 \leqslant z \leqslant 2 P /(d e)} \sum_{1 \leqslant h \leqslant H d^{k-1} e^{-1}} \sum_{M / d<u \leqslant M R / d} e\left(\alpha \Psi_{1}(z, h, u)\right),
$$

and

$$
\Psi_{1}(z, h, u)=u^{-k}\left(\left(z+h u^{k}\right)^{k}-\left(z-h u^{k}\right)^{k}\right) .
$$

But on considering the underlying diophantine equations, the argument of the proof of case (Ia) of [13, Theorem 3.4] provides the upper bound

$$
\begin{aligned}
\int_{0}^{1}\left|F_{d, e}(\alpha)\right|^{4} d \alpha \ll & P^{\varepsilon}\left((P /(d e))^{2}\left(H d^{k-1} e^{-1}\right)^{3}(M R / d)^{3}\right. \\
& \left.+(P /(d e))^{5 / 3}\left(H d^{k-1} e^{-1}\right)^{3}(M R / d)^{4}\right)
\end{aligned}
$$

whence

$$
\sum_{1 \leqslant e \leqslant P} \int_{0}^{1}\left|F_{d, e}(\alpha)\right|^{4} d \alpha \ll d^{3 k-8} P^{\varepsilon}\left(P^{2} H^{3}(M R)^{3}+P^{5 / 3} H^{3}(M R)^{4}\right) .
$$

But our hypothesis that $0 \leqslant \phi \leqslant 1 / k$ ensures that $M R \ll P^{1 / 3}$. Then on recalling our conventions concerning $\varepsilon$ and $R$, we find that the desired upper bound (6) does indeed hold, with $A=3 k-8$. This completes our justification of the aforementioned refinement. 
We now return to the equation (5), observing that the definitions (4) imply that our choice for $\phi$ should be given by $\phi=\min \{\theta, 1 / k\}$, where $\theta$ is defined as in the statement of the lemma. We may now mimic the proof of [15, Theorem 1.1] in order to conclude that

$$
\mu_{s}^{*}=\mu_{u}(1-\theta)+t+u \theta
$$

is permissible, and the conclusion of the lemma follows immediately.

In our application of Lemma 1, we make use of a special case in which certain simplifications are possible. It is convenient to record this consequence of the lemma in the following form.

Lemma 2. Let $k$ be an integer with $k \geqslant 3$, and let $h$ and $\xi$ be positive numbers with $\xi \leqslant 1 / 2$ and $h(1-\xi) \leqslant 4 \xi$. Then, whenever $\delta_{4+h}$ is an associated exponent, one has that $\delta_{4+h(1-\xi)}$ is also associated, where

$$
\delta_{4+h(1-\xi)}=\frac{2(1-\xi)^{2}}{(k+1) \xi}(1+h / 4) \delta_{4+h} .
$$

Proof. We apply Lemma 1 with $u=(4+h)(1-\xi), t=2 \xi$ and $v=4+h$. Since $h(1-\xi) \leqslant 4 \xi$, one has $u \leqslant 4$, and hence the exponent $\mu_{u}=u / 2$ is permissible. Since also the exponent $\mu_{v}=v / 2+\delta_{4+h}$ is permissible, we find from Lemma 1 that the exponent

$$
\mu_{4+h(1-\xi)}=\mu_{u}(1-\theta)+t+u \theta
$$

is permissible, where

$$
\theta=\frac{(1-\xi) \delta_{4+h}}{\xi(k+1)+(1-\xi) \delta_{4+h}} \leqslant \frac{(1-\xi) \delta_{4+h}}{\xi(k+1)} .
$$

We therefore deduce that the exponent $\delta_{4+h(1-\xi)}$ is associated, where

$$
\delta_{4+h(1-\xi)}=\frac{u \theta}{2} \leqslant \frac{(1-\xi)^{2}}{(k+1) \xi}(2+h / 2) \delta_{4+h} .
$$

The conclusion of the lemma follows immediately.

\section{Quasi-Diagonal Behaviour}

We now exploit the recursion formula provided by Lemma 2 in order to establish the quasi-diagonal behaviour exhibited in Theorems 1,2 and 3. There are several possible approaches to this objective, and we concentrate here on simple analyses significant mostly for associated exponents $\delta_{s}$ with $s$ close to 4 . We begin by manipulating the conclusion of Lemma 2 into a form more amenable to our subsequent discussions.

Lemma 3. Let $k$ be an integer with $k \geqslant 3$, and let $\sigma_{0}, \sigma_{1}$ and $\xi$ be positive numbers with $\xi \leqslant 1 / 2$ and $\sigma_{1} \leqslant \sigma_{0}(1-\xi) \leqslant 4 \xi$. Then, whenever $\delta_{4+\sigma_{0}}$ is an associated exponent, one has that $\delta_{4+\sigma_{1}}$ is also associated, where

$$
\delta_{4+\sigma_{1}}=e^{2}\left(\frac{2(1-\xi)^{2}}{(k+1) \xi}\right)^{N-1} \delta_{4+\sigma_{0}}
$$


and

$$
N=\frac{\log \left(\sigma_{1} / \sigma_{0}\right)}{\log (1-\xi)}
$$

Proof. We apply Lemma 2 successively with $h=\sigma_{0}(1-\xi)^{i}$ for $i=0$, $1, \ldots,[N]-1$. In this way we deduce that the exponents

$$
\delta_{4+\sigma_{0}(1-\xi)^{i+1}}=\frac{2(1-\xi)^{2}}{(k+1) \xi}\left(1+\sigma_{0}(1-\xi)^{i} / 4\right) \delta_{4+\sigma_{0}(1-\xi)^{i}}
$$

are associated, whence also we have the associated exponent

$$
\delta_{4+\sigma_{0}(1-\xi)^{[N]}}=C_{N}\left(\frac{2(1-\xi)^{2}}{(k+1) \xi}\right)^{[N]} \delta_{4+\sigma_{0}},
$$

where we have written

But

$$
C_{N}=\prod_{i=0}^{[N]-1}\left(1+\sigma_{0}(1-\xi)^{i} / 4\right)
$$

$$
\log C_{N} \leqslant \sum_{i=0}^{\infty} \log \left(1+\sigma_{0}(1-\xi)^{i} / 4\right) \leqslant \frac{\sigma_{0}}{4} \sum_{i=0}^{\infty}(1-\xi)^{i}=\frac{\sigma_{0}}{4 \xi},
$$

so that in view of our hypotheses concerning $\sigma_{0}$ and $\xi$, it follows that

$$
C_{N} \leqslant e^{\sigma_{0} /(4 \xi)} \leqslant e^{1 /(1-\xi)} \leqslant e^{2} .
$$

Also, one has $[N] \geqslant N-1$ and $\sigma_{0}(1-\xi)^{N}=\sigma_{1}$, and thus we deduce from (7) that the exponent $\delta_{4+\sigma_{1}}$ is associated, where

$$
\delta_{4+\sigma_{1}} \leqslant \delta_{4+\sigma_{0}(1-\xi)^{[N]}} \leqslant e^{2}\left(\frac{2(1-\xi)^{2}}{(k+1) \xi}\right)^{N-1} \delta_{4+\sigma_{0}} .
$$

This completes the proof of the lemma.

The proof of Theorem 1. Suppose that $k \geqslant 60$ and $0<\sigma \leqslant 8 e /(k+1)$. We apply Lemma 3 with $\xi=2 e /(k+1)$ and $\sigma_{0}=8 e /(k+1-2 e)$. Thus we find that the exponent $\delta_{4+\sigma}$ is associated, where

and

$$
\delta_{4+\sigma}=e^{2}\left((1-\xi)^{2} / e\right)^{N-1} \delta_{4+\sigma_{0}}
$$

$$
N=(\log (1-\xi))^{-1} \log \left(\frac{\sigma(k+1-2 e)}{8 e}\right) .
$$

But if $\delta_{5}$ is an associated exponent, then there is no loss in supposing that $\delta_{4+\sigma_{0}} \leqslant \delta_{5}$. Also, one has

$$
-\log (1-\xi)=\sum_{n=1}^{\infty} \frac{\xi^{n}}{n} \leqslant \frac{\xi}{1-\xi}=\frac{2 e}{k+1-2 e},
$$


and when $k \geqslant 60$, one has

$$
\frac{k+1-2 e}{8 e} \geqslant \frac{k}{24}
$$

Thus we deduce that

$$
\begin{aligned}
\delta_{4+\sigma} & \leqslant \frac{e^{3}}{(1-\xi)^{2}}\left(\frac{\sigma k}{24}\right)^{2-1 / \log (1-\xi)} \delta_{5} \\
& \leqslant e^{3}(1-2 e / 61)^{-2}\left(\frac{\sigma k}{24}\right)^{1+(k+1) /(2 e)} \delta_{5} .
\end{aligned}
$$

The conclusion of Theorem 1 therefore follows with a modicum of computation, on noting that Theorem 1.3 of [15] supplies the associated exponent

$$
\delta_{5}=\frac{8 k^{1 / 2}}{5 e} \exp \left(-\frac{16 k}{25 e^{2}}\right) \text {. }
$$

The proof of Theorem 2. Suppose that $k \geqslant 3$ and that $\sigma_{0}$ and $\sigma_{1}$ satisfy the hypotheses of the statement of Theorem 2. We apply Lemma 3 with $\xi=\sigma_{0} /\left(4+\sigma_{0}\right)$, deducing that the exponent $\delta_{4+\sigma_{1}}$ is associated, where

$$
\delta_{4+\sigma_{1}}=e^{2}\left(\frac{(k+1) \xi}{2(1-\xi)^{2}}\right)\left(\frac{\sigma_{1}}{\sigma_{0}}\right)^{\kappa} \delta_{4+\sigma_{0}}
$$

and

$$
\kappa=2+\frac{\log ((k+1) \xi / 2)}{\log (1 /(1-\xi))}
$$

But

$$
-\log (1-\xi) \leqslant \xi /(1-\xi)=\sigma_{0} / 4,
$$

whence the upper bound $\sigma_{0} \leqslant 4$ leads to the lower bound

$$
\kappa \geqslant 2+\frac{4}{\sigma_{0}} \log \left(\frac{(k+1) \sigma_{0}}{2\left(4+\sigma_{0}\right)}\right) \geqslant 2+\frac{4}{\sigma_{0}} \log \left(\frac{(k+1) \sigma_{0}}{16}\right) .
$$

Also,

$$
\frac{\xi}{2(1-\xi)^{2}} \leqslant \frac{\sigma_{0}}{2\left(4+\sigma_{0}\right)}\left(\frac{4}{4+\sigma_{0}}\right)^{-2}=\frac{\left(4+\sigma_{0}\right) \sigma_{0}}{32} \leqslant 1 .
$$

On collecting together the above inequalities, we conclude that

$$
\delta_{4+\sigma_{1}} \leqslant e^{2}(k+1)\left(\frac{\sigma_{1}}{\sigma_{0}}\right)^{2+\frac{4}{\sigma_{0}} \log \left((k+1) \sigma_{0} / 16\right)} \delta_{4+\sigma_{0}},
$$

and the conclusion of Theorem 2 follows immediately.

The proof of Theorem 3. We apply Lemma 3 with $\xi$ defined as in the statement of Theorem 3, this choice having been determined by an optimisation, the details 
of which we may suppress. Thus, when $\sigma_{0}$ and $\sigma_{1}$ satisfy the hypotheses of the statement of the theorem, we find that the exponent $\delta_{4+\sigma_{1}}$ is associated, where $\delta_{4+\sigma_{1}}$ is defined by (8) and (9). But in view of the equation defining $\xi$ presented in the statement of Theorem 3, we find that for $k \geqslant 8$, the formula (9) becomes

$$
\kappa=2+(1-\xi) / \xi=1+1 / \xi,
$$

whence the upper bound (10) leads from (8) to the estimate

$$
\delta_{4+\sigma_{1}} \leqslant e^{2}(k+1)\left(\sigma_{1} / \sigma_{0}\right)^{1+1 / \xi} \delta_{4+\sigma_{0}} .
$$

This completes the proof of the theorem when $k \geqslant 8$. When $3 \leqslant k \leqslant 7$, meanwhile, we take $\xi=1 / 2$, and on combining (8)-(10) we again arrive at the conclusion claimed in the statement of Theorem 3 .

We note that in our application of Lemma 3, we are restricted in our choice of $\xi$ to the interval $0<\xi \leqslant 1 / 2$. Our decision to take $\xi(k)=1 / 2$ for $3 \leqslant k \leqslant 7$ is consequently determined by the observation that under the latter circumstances, the solution of the equation otherwise defining $\xi$ exceeds $1 / 2$.

\section{References}

[1] Baker RC, Brüdern J, Wooley TD (1995) Cubic diophantine inequalities. Mathematika 42: 264-277

[2] Brüdern J, Kawada K, Wooley TD, Additive representation in thin sequences (submitted)

[3] Brüdern J, Wooley TD (1999) On Waring's problem: a square, four cubes and a biquadrate. Math Proc Cambridge Philos Soc 127: 193-200

[4] Brüdern J, Wooley TD (2000) On Waring's problem for cubes and smooth Weyl sums Proc London Math Soc (to appear)

[5] Brüdern J, Wooley TD (2000) On Waring's problem: three cubes and a sixth power Nagoya Math J (to appear)

[6] Brüdern J, Wooley TD (2000) On Waring's problem: two cubes and seven biquadrates Tsukuba J Math (to appear)

[7] Heath-Brown DR (1997) The density of rational points on cubic surfaces. Acta Arith 79: 17-30

[8] Hooley C (1981) On another sieve method and the numbers that are a sum of two $h$ th powers. Proc London Math Soc. (3) 43: 73-109

[9] Hooley C (1996) On another sieve method and the numbers that are a sum of two $h$ th powers. II. J Reine Angew Math 475: 55-75

[10] Skinner CM, Wooley TD (1995) Sums of two kth powers. J Reine Angew Math 462: 57-68

[11] Vaughan RC (1989) A new iterative method in Waring's problem. Acta Math 162: 1-71

[12] Vaughan RC (1989) A new iterative method in Waring's problem II. J London Math Soc (2) 39: 219-230

[13] Vaughan RC, Wooley TD, Further improvements in Waring's problem, IV: higher powers Acta Arith (to appear)

[14] Wooley TD (1994) Quasi-diagonal behaviour in certain mean value theorems of additive number theory. J Amer Math Soc 7: 221-245

[15] Wooley TD (1995) Breaking classical convexity in Waring's problem: sums of cubes and quasidiagonal behaviour. Invent Math 122: 421-451

Author's address: TD Wooley, Department of Mathematics, University of Michigan, East Hall, 525 East University Avenue, Ann Arbor, Michigan 48109-1109, U.S.A., e-mail: wooley@math.lsa.umich.edu 